\title{
Quaderni
}

QUADERNI Communication, technologies, pouvoir

89 | Hiver 2015-2016

Penser l'avenir : le CESTA, un think tank atypique

\section{Le CESTA : une autre conception de l'innovation et de la prospective}

\section{Marc Chopplet}

\section{(2) OpenEdition}

1 Journals

\section{Édition électronique}

URL : http://journals.openedition.org/quaderni/953

DOI : 10.4000/quaderni.953

ISSN : 2105-2956

\section{Éditeur}

Les éditions de la Maison des sciences de l'Homme

\section{Édition imprimée}

Date de publication : 5 janvier 2016

Pagination : 25-42

Référence électronique

Marc Chopplet, «Le CESTA : une autre conception de l'innovation et de la prospective », Quaderni [En ligne], 89 | Hiver 2015-2016, mis en ligne le 05 janvier 2018, consulté le 20 avril 2019. URL : http:// journals.openedition.org/quaderni/953 ; DOI : 10.4000/quaderni.953 


\section{$D$ ossier}

\section{Le CESTA : \\ une autre \\ conception \\ de l'innovation et \\ de la prospective}

\author{
Marc \\ Chopplet
}

Fellow IEA Nantes
Affaire des Futures Studies ou de notes de conjoncture, la prospective, à l'exception de quelques grands organismes, est surtout devenue en France en 2016 un outil au service du marketing de nouveaux produits pour des entreprises mondialisées ${ }^{1}$, de la spéculation des marchés financiers ou de scénarii catastrophes prenant en compte de nouveaux indicateurs prévisionnels comme le réchauffement climatique. Ces approches finalement très pauvres, prises entre spéculation planétaire et catastrophe planétaire, donnent le sentiment inquiétant d'un avenir éminemment dangereux et borné. Vision manichéenne fortement surdéterminée où les marges de manœuvre du politique sont faibles faisant ressembler les politiques de gauche à des politiques de droite et inversement.

Il n'en fut pas toujours ainsi et la prospective a pu jouer un rôle essentiel dans d'autres visions du monde et de l'innovation, plus ouvertes, plus volontaires et plus optimistes. Cette histoire est aujourd'hui largement oubliée. Cet article s'attachera à analyser les formes, circonstances et objectifs du développement de la prospective en France et à dégager l'apport original d'Yves Stourdzé, sociologue des organisations et de la communication, et du Centre d'Études des Systèmes et des Technologies Avancées (CESTA) qu'il a dirigé de 1982 à 1986. Organisme qui fut peut-être la dernière initiative d'État d'une politique publique de cette ampleur au service du développement de ce qu'on appelait alors « les nouvelles technologies » ou les «technologies avancées ». Nous retracerons rapidement dans un premier temps les attendus, rôles et apories de la prospective des années 1960-1980 avant d'analyser les apports d'Yves Stourdzé et du CESTA. 


\section{Prospective et politique. Une démarche au service de la modernisation}

En France, les années 1960-1980 sont travaillées par la prospective. Mouvement venu des ÉtatsUnis et des travaux de la Rand Corporation ${ }^{2}$ au lendemain de la Seconde Guerre mondiale, elle est au cœur de réflexions convergentes d'acteurs aux intérêts pourtant très divers au sein de la société française. Gaston Berger, haut commis de l'État au ministère de l'Éducation Nationale, en créant en 1957 le Centre d'études prospectives apparaît comme le précurseur de ces travaux.

Elle repose sur deux constats, fondements de l'ensemble de la démarche. D'une part, on observe une accélération des changements économiques, technologiques et sociaux qui imposent un autre regard sur l'avenir ; d'autre part cet avenir se construira sur des ruptures. Conséquence essentielle : les instruments de prévision regardant le passé pour inférer des tendances à venir sont inadaptés à l'identification des " changements secrets » ou « signaux faibles » qui craquellent dès aujourd'hui notre quotidien.

Ces deux éléments : accélération des changements, rupture des continuums temporels d'une histoire sociopolitique au long cours sont repris par Bertrand de Jouvenel et la revue Futuribles à partir de 1961. Ils constituent la base de travaux visant à élaborer des scénarii sur l'évolution de différents secteurs et domaines d'activité. Les démarches et méthodes mises en œuvre sont peu à peu reprises d'abord au niveau national, puis au niveau régional, par l'État dans la préparation d'actions de programmations à court et moyen terme venues, elles aussi, des États-Unis :
Rationalisation des choix budgétaires (RCB), Plan d'Aménagement du Territoire, Prospective des armées, Plan calcul d'informatisation de l'administration... Elles favorisent la création d'instances comme la DATAR ou de Directions nouvelles au sein de différents ministères : Éducation nationale, Économie et Finances, Armée, Équipement... ainsi qu'au niveau régional avec les OREAM ${ }^{3}$.

Dans ce cadre qui est celui de l'État gaullien, la prospective n'est plus seulement une manière large de s'interroger sur l'évolution d'un domaine d'activité, voire de la société, mais une démarche opérationnelle tournée vers la programmation et l'action avec, pour corollaire, une très grande sensibilité aux éléments qui apparaissent, ou peuvent apparaître, comme des résistances et des freins au changement programmé. Elle constitue un moyen pour identifier les résistances possibles dans les scénarios, pour envisager les conséquences essentiellement macro-économiques des programmations envisagées, mais surtout pour convaincre de la crédibilité, mieux de la nécessité, des propositions soumises aux décideurs.

Lucien Sfez, en 1970 dans L'Administration prospective, analyse ce phénomène nouveau qu'il considère comme le "seul phénomène sérieux de l'administration contemporaine 4 ». Il réalise un panorama très complet et documenté des politiques, des organismes et des institutions prospectives publiques et privées, centralisées ou décentralisées en cherchant à caractériser leurs missions, leurs modes de fonctionnement, leurs interactions avec l'administration traditionnelle et les problèmes nouveaux, notamment juridiques, qu'elles posent. Nous retiendrons de son analyse 
deux points qui permettent de caractériser le discours prospectif et de le positionner d'un point de vue politico-institutionnel au sein de la haute administration.

Le premier point est déterminant. Identifier les caractéristiques du discours prospectif, c'est à la fois le distinguer d'autres formes de discours et fonder sa légitimité. Il s'agit en effet d'écarter les critiques encourues par les scénarii accusés d'être de pures fictions, des jeux de l'imagination, voire des utopies sociales ou la simple reformulation de très anciens mythes civilisateurs d'une société idéale. Il s'agit en même temps d'asseoir sa crédibilité. Ce travail d'analyse débouche sur des distinctions fortes. Trois éléments clés sont dégagés de la conception même de la prospective par les prospectivistes :

- Le rapport au temps ${ }^{5}$. Si le mythe et l'utopie apparaissent comme deux moyens pour supporter le présent ; l'un en proposant une explication et une solution s'inscrivant dans un temps originaire, l'autre en proposant une solution dans un temps à venir espéré, le discours prospectif ouvre au contraire le champ des possibles en analysant les contradictions actuelles (proche en cela d'une conception dialectique de l'Histoire) et retient le principe de surdétermination de l'économie sur tout autre facteur comme clé de lecture et de discrimination des scénarii. Il ouvre sur une pluralité de temps dont il se donne les moyens de peser et évaluer la vraisemblance à partir de critères et indicateurs définis par avance sur la base de travaux scientifiques ;

- Le caractère scientifique et critique de la méthode. Le discours prospectif, ce grand récit d'avenir, est fondé « scientifiquement » à la fois dans sa méthode et dans les données qu'il utilise. Il constitue, de ce fait, un élément déterminant de critique du fonctionnement de l'administration traditionnelle et un instrument opérationnel pour l'action et la planification ${ }^{6}$;

- La spécificité de la démarche. Mettant en œuvre des interventions en mode projets, nouvelles dans l'administration, elle encourage et stimule des travaux qui peu à peu dessinent les contours d'un véritable « projet de civilisation ${ }^{7} »$.

Second élément de cette réflexion : sur qui s'appuie cette administration prospective ? Quels en sont les acteurs? Qui la porte ? Si l'enjeu est bien celui de la rationalisation des choix et du développement de stratégies de changement, il est clair que les porteurs doivent présenter une surface d'intervention importante et une légitimité forte. Or, curieusement, "la prospective et les stratégies de développement de celle-ci qui entrainent le bouleversement des structures classiques $^{8} »$ sont moins liées à l'action directe du politique qu'à une transformation interne de l'administration 9 qui créé des organes, des technostructures, à la tête desquelles se trouvent de hauts-fonctionnaires appartenant au parti majoritaire et à ses alliances. Le choix de la prospective est un choix d'appareil politique permettant de passer par-dessus les instances nationales parlementaires, certains ministères techniques et les institutions locales élues ou non. Insérée dans une stratégie de changement se présentant comme «modernisation » et « rationalisation», elle est l'instrument qui permet de court-circuiter divers niveaux de représentations politiques et jeux de pouvoir ${ }^{10}$. 
Crise, rupture et dysfonctionnement. La prospective : une autre manière de comprendre l'Histoire

Cette approche prospective interne à l'administration et orientée vers la planification tient peu compte du développement des nouvelles technologies ${ }^{11}$ non plus que de l'impact qu'eurent les événements de Mai 1968 sur la prise de conscience des ruptures et des mutations de la société française. Préoccupée de modernisation technocratique, les ruptures qu'elle veut anticiper sont celles qu'elle-même créerait par la mise en œuvre de ses travaux. Avec 68 et la crise pétrolière de 1973 c'est une autre vision qui tend à s'imposer où les notions de crise, de rupture, de dysfonctionnement apparaissent déterminantes ${ }^{12}$. Le bilan de dix années d'études de prospective du Laboratoire de conjoncture et prospective réalisé en $1977^{13}$ illustre, du point de vue de la recherche, les questions posées et les transformations de la prospective. En premier lieu celles du champ concerné.

En effet, la définition retenue par le laboratoire met l'accent sur deux points importants. D'une part « la prospective n'est autre qu'une lecture particulière de l'Histoire, celle des projets des hommes et de leurs luttes pour les défendre et les faire aboutir ${ }^{14} »$. D' autre part elle est «fondamentalement liée à une conception des sciences sociales et humaines que le courant né de 68 a canalisé $^{15} \gg$.

Ces deux points introduisent un débat lourd de sens. Le regard porté sur l'avenir n'est pas un regard technique, fruit de discussions d'experts mettant en œuvre divers outils et méthodes prévisionnelles, statistiques ou macro-économiques, mais une démarche conceptuelle qui a à voir avec la manière dont on lit et écrit l'Histoire. Les références relèvent du marxisme mais surtout de la lecture du travail d'historien (et de sociologue) qu'en donne Paul Veyne ${ }^{16}$ et de la méthode progressive-régressive de Jean-Paul Sartre ${ }^{17}$ dans sa définition de l'homme comme projet et son rapport à l'Histoire.

L'objet de la prospective, ambition d'une toute autre envergure, est de comprendre finement les projets au sens de Sartre pour les insérer dans « la globalité de la réalité sociale en mouvement ${ }^{18} »$. C'est à la fois une critique implicite des travaux de prospective des années 60 qui ne sont finalement que de la prévision pour l'action; c'est-à-dire « un instrument de gestion du système économique ${ }^{19} \gg$ qui opère dans un espace sans Histoire, mais aussi une réflexion de fond sur le travail de prospective qui concerne le futur de la société ou d'un ensemble sociétal.

La critique fondamentale est celle des critères et indicateurs retenus. C'est-à-dire de la scientificité de la démarche adoptée jusqu'à présent. Qu'est-ce qui fait sens ? Que faut-il prendre en compte ? Les contradictions ? Les discontinuités? Les crises ? Ces notions n'appellent-elles pas inévitablement une démarche volontariste qui jette le doute sur l'objectivité scientifique des scénarios produits ${ }^{20}$ ? Quant au principe de surdétermination économique, ne devient-il pas très rapidement l'unique déterminant; alors même que l'économie peut ne pas être, au présent, un déterminant d'actions ou de réactions ? Mai 1968 ne démontre-t-il pas que la prévision économique peut être un «leurre $e^{21} »$ ? 
Cela conduit à déplacer le centre de gravité de la prospective. Fonder une science de la prospective, une « prospectologie », c'est d'abord travailler sur le présent comme espace-temps du rapport de l'homme à l'Histoire. Le présent est le lieu et le moment du changement ; c'est une porte de l'avenir que l'avenir, précisément, permettra d'expliquer rétrospectivement. Pour reprendre la formule de Jean-Paul Sartre, «l'homme est pour lui-même et pour les autres un être signifiant puisqu'on ne peut jamais comprendre le moindre de ses gestes sans dépasser le présent pur et l'expliquer par l'avenir ${ }^{22} »$ ou encore comme le déclare Christian Goux, "l'avenir ; clé d'explication du présent ». Le présent c'est l'avenir déjà là, non au sens de tendances, de pentes, d'orientations, de signaux faibles mais au sens de totalité dynamique de projets, de structures des formations sociales à reconnaître et à intégrer dans leur nature profondément contradictoire. La notion de conflit - et non simplement de contradiction - est l'élément central de la prospective et la prospective du changement social rend compte « de la nécessité de renverser la problématique usuelle... pour rendre compte des formations sociales en situation de rupture ${ }^{23} »$. Sur cette pente il y a moins une vérité explicative d'un tout qu'un foisonnement, une multiplicité de temporalités diverses à identifier, explorer, analyser et non plus à canaliser ou discriminer.

Sur le volet technologie, le constat est celui de son émergence et ceci pour plusieurs raisons. La première est pratique : sa croissance est mesurable et à ce titre elle constitue un invariant facile à suivre. La seconde, qui dérive de la première, c'est qu'elle donne lieu à un modèle « population-investissement » (repris par le Club de Rome) faisant du même coup de la production technologique un modèle industriel, économique et d'organisation du travail et donc un facteur décisif des relations sociales au détriment de tout autre indicateur. Or, c'est là le danger et, pour bien faire, il faudrait inverser le regard, "passer de l'autre côté du miroir » et envisager la technologie «dans le mode de relations sociales auquel et duquel elle participe ». C'est l'appropriation de la technologie qui est signifiante et non sa production : «la technologie est une production sociale, dont les rapports sociaux générés par la contrainte de la mise en valeur, sont les déterminants $^{24} \gg$.

\section{Ce qu'attendent les pouvoirs publics à travers la création du CESTA}

Le rapport remis au Premier ministre en décembre 1981 pour la création du Centre d'Études des Systèmes et des Technologies Avancées (CES$\mathrm{TA})^{25}$ est clairement centré sur le développement des nouvelles technologies sur la base de ce qui est présenté comme trois grandes évidences :

1. Le schéma de développement des pays industrialisés place la technologie comme pôle majeur des nouveaux rapports entre État - industrie et recherche ;

2. Ce schéma « recherche - innovation technologique - progrès industriel - croissance économique - bonheur » fait débat : quelle technologie pour quels besoins sociaux ? La technologie est, en ce sens, indissociablement à la fois un enjeu stratégique et un enjeu politique ${ }^{26}$;

3. Un effort pluridisciplinaire est à réaliser pour rapprocher culture scientifique et culture humaniste, évaluer, promouvoir, former, informer et 
sensibiliser en vue de "l'enrichissement d'une culture capable de sous-tendre un projet de société27 ${ }^{27}$.

Les deux premiers points s'inscrivent dans le droit fil des rapports de Simon Nora et Alain Minc sur l'informatisation de la société et de François Gros, François Jacob et Pierre Royer sur les Sciences de la vie et la société ${ }^{8}$. Le dernier point est plus spécifique et plonge ses racines notamment dans les travaux de Jacques Attali et Marc Guillaume et se nourrit de l'apport du Groupe des $\operatorname{Dix}^{29}$. Dans ce projet qui vise à contribuer « au développement et à la diffusion des technologies, au plan national et international, tout en analysant et préparant les conditions de leur insertion dans les structures socio-économiques et socioculturelles ${ }^{30} »$, la prospective ne constitue pas l'élément central. Elle en est une conséquence dérivée en termes de méthode et d'approche stratégique. Il s'agit d'assurer une veille internationale sur les nouvelles recherches et technologies émergentes; de les passer au crible d'une analyse « stratégique » en termes de forces, faiblesses, opportunité et menaces ${ }^{31}$ pour la France et d'en mesurer l'impact sur la sécurité, l'emploi et l'environnement. Le modèle proposé, là encore, est américain : celui de l'Office of Technology Assement ${ }^{32}$ créé en 1972 par le Congrès américain avec pour mission de fournir des analyses « objectives » dans les domaines complexes scientifiques et technologiques de la fin du $\mathrm{XX}^{\mathrm{e}}$ siècle.

Avec un nouvel objet, celui du développement d'une culture technique comme projet de civilisation, et une problématique qui ne relève plus d'une planification volontariste, le projet reste assez proche cependant de celui de l'administration prospective des années 60-70. On se situe, en terme de conception, dans le cadre de ce que Pierre Musso ${ }^{33}$ identifiait comme «l'État savant et ingénieur " créant les conditions d'acceptabilité, d'appropriation et de diffusion des technologies. Fait nouveau toutefois, les sciences humaines et sociales y sont convoquées pour leur vertu (supposée) d'aide à la définition des conditions appropriées à un dialogue avec la société civile, de prise en compte des besoins des utilisateurs et de création d'un climat favorable à l'émergence douce d'une culture technique. Cette vertu lénifiante pouvant être recherchée à travers des « expérimentations techniques ou sociales» qui permettent d'ajuster les modes opératoires et les moyens.

Le rapport, rédigé avec les contraintes propres d'un rapport destiné à des décideurs politiques, prépare les décisions qui seront annoncées à l'issue du Colloque national Recherche et Technologie en janvier 1982.

\section{Yves Stourdzé et la prospective}

Les références d'Yves Stourdzé, sociologue des organisations et de la communication, appelé à prendre la direction du CESTA, sont d'une toute autre nature. D'un côté elles s'ancrent tout particulièrement dans une lecture de l'histoire des luttes sociales et politiques; et de l'autre dans la vision aiguë de l'histoire des heurs et malheurs de l'innovation technologique et de sa captation par les forces militaro-industrielles en France. Acteur engagé dans le mouvement du 22 mars en 1968, son approche est critique du système français et de ses scléroses : la rupture n'est pas à venir ; 
elle a d'ores et déjà eu lieu. Il s'agit à présent de faire comprendre cet événement majeur et d'agir au sein de ce nouvel espace-temps.

On n'assiste pas à une rupture ou à une crise dont il s'agit de faire le diagnostic « à chaud » pour conjecturer les développements (démarche analytique et cognitive), mais de mettre en œuvre opérationnelle les fruits d'une réflexion (démarche stratégique) qui a d'ores et déjà identifiée que nous marchons sur les ruines de la société industrielle ${ }^{34}$. L'histoire est déjà faite et ce qu'il faut c'est repérer les nouveaux groupes humains, les nouvelles communautés agissantes et œuvrer pour qu'elles agissent plus encore. La culture est déjà là : elle est cybernétique. Elle refuse le report de jouissance mis en œuvre par les organisations aux objectifs planifiés ${ }^{35}$ pour inviter à une parole libérée. Il faut la reconnaître et la faire vivre. D'une certaine manière la prospective est déjà faite : il s'agit soit d'une prospective de risques de blocages liés à l'émergence de pratiques nouvelles (études réalisées par Yves Stourdzé en Bretagne et en Nord-Pas-de-Calais pour le compte de la Direction à la prospective du ministère de l'Équipement ${ }^{36}$ ), soit d'une lecture nouvelle faisant entrer le grand vent de l'Histoire, en particulier des sciences et des technologies grand public, dans son orbite.

Posons Les Ruines du futur comme un ouvrage de prospective au sens large abordant aussi bien les questions de l'urbain, des transports, de la science et de la technologie que les relations humaines. Un ouvrage d'avenir pour explorer le présent. La première phrase : «nous nous promenons aujourd'hui dans les ruines de notre avenir » disqualifie à la fois tous les produits du présent qui ne sont que ruines, et elle nous donne ces ruines toutes entières comme horizon d'avenir. Il s'agit moins d'une conception pessimiste que d'une véritable remise en perspective de la prospective où l'essentiel n'est pas dans les artefacts périssables et les simulacres déjà balayés mais dans les temps et les espaces nouveaux de liberté où «se mettent en branle une multitude de dissidences ». Les plans, les bornes sont dès lors « incompréhensibles » car inactuels et en porte à faux. Ils ne servent à rien ; pas plus que le critère de choix de l'économique « dernière instance » désormais " grotesque $»^{37}$. La prospective d'hier servait les intérêts stérilisant des organisations tentaculaires et mortifères. Elle visait à canaliser et bloquer les énergies. Une prospective nouvelle s'annonce. Exprimée en termes nouveaux, lançant éclairs et tonnerres, annonçant ruines et catastrophes, rejetant le sacrifice, libérant la parole aux multiples accents et attendant tout, le meilleur et le pire, des nouveaux usages proliférant sur la toile, elle balaie toutes prospectives antérieures. Le ton est prophétique, mais précisément la prophétie est par avance disqualifiée ${ }^{38}$, sauf à reconnaître comme le fait Jacques Attali que le prophète de malheur est le meilleur conseiller du pouvoir ${ }^{39}$. Il ne s'agit pas non plus d'utopie (ni d'ailleurs d'une dystopie) mais d'une prospective tendue comme un arc vers un avenir encore à peine dicible.

Il y a de l'espoir dans cette vision. De l'espoir après et dans la catastrophe malgré les multiples types de recyclages mis en œuvre par les organisations pour perdurer car elles ne parviendront pas à bloquer ce qui « entre le temps passé et le temps à venir suinte »; c'est-à-dire le présent, « l'insolite d'une rencontre, d'une dissidence, 
d'une variation ${ }^{40} »$. Le constat est définitif et assuré : " une transformation radicale a fait basculer le système à son insu - le plaçant en position de déliquescence : le monde instrumental, l'univers anodin et neutroïde de la consommation s'est transmué en espace pervers de la gestion démultipliée : bricolage de condensés implosifs. Partout, pulvérisés, des instruments d'annulation ${ }^{41} "$; ces instruments d'annulation que sont les organisations qui figeaient le temps, l'arrêtaient, le dévoraient comme Saturne ses enfants. La roue du temps; un temps cyclique comme la sphère d'Empédocle (fragment 161), celle du pouvoir, peut se remettre en marche ${ }^{42}$.

On mesure dans ce travail la distance par rapport à sa thèse rédigée en 1973 portant sur l'organisation qui faisait le constat terrible d'un enfermement dominé par les figures tutélaires de Taylor pour l'organisation industrielle, Lénine pour l'organisation politique et Clausewitz pour l'organisation militaire ${ }^{43}$. Face à elle l'anti-organisation, effervescente et explosive, multiforme et variée se révélait « vulnérable $»^{44}$ et impuissante. Il s'agissait alors d'inventer « un temps et un espace sur lesquels l'organisation ne puisse avoir de prise ${ }^{45} »$. Cinq ans plus tard le ton a changé : «l'œil classique s'embue et le brouillard envahit le panopticon: c'est que l'espace se tord et se mettent à vibrer les plasmas instables, les pièges, les vides. Stratégie de mise à $s a c^{46} »$. À la révolution se sont substitués «le saut des puces électroniques » et «l'insurrection dissidente des microprocesseurs ${ }^{47} »$.

En fait, ce qui a profondément changé c'est le regard porté sur ce que cette organisation tentaculaire et dominante a fait des innovations technologiques et comment elle les a mises en œuvre. Ce travail s'est effectué par un retour aux origines, par la mobilisation d'une démarche généalogique pour appréhender morceau par morceau, brique par brique, de l'invention aux choix et déterminants de la mise en œuvre opérationnelle, les tours et détours de l'appropriation politique, économique et sociale des technologies dans le domaine de la communication (mais pas uniquement) qui révèlent les failles de ce système organisationnel. La découverte d'une préférence pour des équipements $«$ lourds $»^{48}$ sur lesquels un contrôle fort puisse s'exercer est à mettre en parallèle avec le témoignage que Marc Bloch consacrait à « l'étrange défaite » de l'armée française en 1940 ; procès terrible de l'aveuglement, de l'ignorance, de l'entêtement et de la « complaisance envers soi-même $e^{49}$ » qui, au-delà du commandement militaire, stigmatisait la société et la communauté politique française.

La crise, la rupture ne sont rien. On s'en effraie ou on s'en réjouit comme d'une possibilité de renouveau alors même qu'il y a une liaison fondamentale et étroite entre pouvoir et crise où la crise constitue un instrument permettant de briser les résistances ${ }^{50}$. C'est donc moins la crise, la rupture apparente, l'irruption, la contradiction qui sont signifiants dans une approche prospective mais les traces infimes, les glissements, les déliquescences, tous ces mouvements souterrains à peine perceptibles qui font vibrer les frontières et mettent à mal les édifices les plus solides, longtemps avant qu'ils ne s'écroulent effectivement. Tout est signe et fait signe : «l'anti-économie, l'anti-CEdipe, la "non-bataille » autant de symptômes que les thématiques austères de l'organisation, de l'institution et de l'économie 
ont fait leur temps ${ }^{51} »$.

Cette prospective délivre une conception nouvelle de l'innovation comme exploration du libre. Par rapport aux travaux du Laboratoire de conjoncture et prospective embarrassés dans une totalité dynamique de projets et le décorticage fin de rapports sociaux, Stourdzé opère un double mouvement : si les nouveaux usages font éclater les systèmes ${ }^{52}$ et mettent à mal des planifications mortifères et des organisations sacrificielles, ce qui est essentiel c'est surtout la charge de critique qu'ils véhiculent et qui donne un sens à l'Histoire (il ne s'agit pas simplement d'attendre que l'Histoire s'écrive dans l'enchevêtrement meurtrier des projets et des formations sociales comme une loi empirique de rapports de force ou de sélection naturelle d'où émergerait l'avenir) et les innovations qui contribuent à ce mouvement, le poussent, l'entraînent, l'accélèrent. Or ces innovations, à n'en pas douter viennent de ce qui échappe aux organisations et aux planifications : à savoir les découvertes scientifiques : hier l'électricité, aujourd'hui le microprocesseur. Ainsi, s'il y a une prospective ce doit être celle de la recherche scientifique et de ses découvertes à l'échelle du nanomètre comme l'ADN ou de la gravure des composants élémentaires sur la puce de silicium : nouveaux lieux où s'inventent des énergies puissantes et libres et où s'inscrit le neuf.

\section{Le CESTA}

En forçant le trait on pourrait dire que tout, ou presque, a été dit de la prospective au CESTA dès Les Ruines du futur. L'essentiel, en retour, est ailleurs : il est dans l'organisation. C'est l'organisation qui doit innover et être prospective. C'est là que gît la question du pouvoir et l'invention d'un autre avenir. En effet, si « le monde qui se dévoile progressivement devant nous est l'inverse d'un univers monochrome et stable... les hommes qui y naviguent doivent posséder le sens du réseau, de la connexion, de la négociation. La variété, la diversité, la multiplicité ne doivent plus être considérées comme des intrus, mais comme des signes de vitalité et de puissance. C'est pourquoi le management horizontal, stratégique et polycentrique se substitue aux formes de commandement classiques jonchées sur des structures verticales ». Le CESTA sera cette « organisation de « demain » mettant en œuvre « la vertu de schémas cellulaires et vivants qui forment la trame solide d'un développement cohérent au sein d'un univers instable et dangereux, en remplacement de modèles mécaniques voués, en raison même de leur absence d'adaptation à la disparition ${ }^{53} \gg$. Dans Organisation, Antiorganisation, Yves Stourdzé s'interrogeait sur la possibilité d'inventer un temps et un espace sur lesquels l'organisation ne puisse avoir prise ${ }^{54}$. Le CESTA sera pour lui et les équipes qu'il constituera autour de lui une tentative, consciente ou inconsciente, d'instauration d'un tel espace-temps de la recherche, de l'innovation et de nouvelles interactions entre les acteurs, entre les disciplines, entre les continents.

La prospective pour l'administration prospective était une manière de modéliser l'avenir, d'en tracer les routes, d'en organiser la gestion, d'en préparer le management. Les objectifs étaient ceux de la rationalisation et de la modernisation.

La prospective pour le laboratoire de conjoncture était avant tout une démarche méthodologique 
et une nouvelle science visant à comprendre l'évolution de formations sociales et à dégager d'une part des tendances générales (temps long) et d'autre part une analyse-diagnostic (temps court) pouvant déboucher sur des scénarios ${ }^{55}$. Il s'agissait d'une démarche composite au service d'une approche cognitive.

Si le livre blanc de la création du CESTA reprend des objectifs de l'administration prospective - et d'ailleurs à la même époque d'autres instances sont créées avec des objectifs similaires de programmation et de planification dans le domaine de la recherche - il introduit également une préoccupation sociale forte, une exigence de conduite du changement en termes d'animation, de formation, de sensibilisation et de contrôle des risques au service d'un projet de société à construire au plan national et international.

Or, à ces positions institutionnelles Yves Stourdzé répond en proposant une démarche opérationnelle reposant sur trois piliers :

- une lecture de l'histoire par un recours à la généalogie qui permet de décrypter le présent. Que disent les appropriations passées des technologies et que révèlent les analyses fines et les lectures attentives de mondes en gestation sur ce que nous devons faire pour éviter de retomber dans les ornières des blocages et des enfermements paranoïaques qui ont conduit à des catastrophes et aux ruines sur lesquelles nous marchons?

- un principe de discrimination pour lire dans le présent les formes d'avenir en repérant les implosions souterraines et les libertés émergentes de paroles libérées ;
- l'intuition que les discours permettant de faire entendre ces bruissements sont ceux qui font appel à l'imagination, aux images et aux symboles ; ni discours prophétique ni construction utopique mais un nouvel espace libre, poétique, vivant, foisonnant et multiforme

Cette approche a plusieurs conséquences fortes au plan de l'organisation et au plan de l'action qui la distinguent d'autres démarches reposant sur la prévision :

1. Si elle s'appuie sur des recensions, des synthèses et des analyses d'états des sciences et technologies (de nombreux rapports et ouvrages édités par le CESTA sur les biotechnologies, les matériaux, la robotique, les didacticiels l'attestent) c'est moins pour en inférer des développements technologiques futurs que comme base d'analyse de potentialités et de projections de ces potentialités dans de nouveaux usages. Cognitiva, qui donne lieu à l'organisation d'un colloque en 1985 intitulé $A$ la frontière de l'intelligence artificielle, des sciences de la connaissance, des neurosciences ${ }^{56}$, c'est-à-dire l'alliance de l'intelligence artificielle, de l'imagerie et de la neurobiologie, est un bon exemple de cette projection qui consiste à réunir les fragments d'un puzzle extraordinairement éclaté. L'interrogation sur la mise en œuvre des technologies dans des milieux extrêmes qui donne lieu également à l'organisation d'un colloque en $1986^{57}$ est de même nature ; de même que les deux colloques organisées sur l'Image (Biarritz 21-25 mai 1984 et Nice 21-25 avril 1986). On pourrait multiplier les exemples. L'IPSPT (Institut de prospective scientifique et de prévision technologique) un moment envisagé déplace ainsi clairement la prospective vers la 
science et laisse la technologie, fille de la science, à la prévision. C'est bien à une prospective des sciences qu'il faut se livrer et laisser jouer les expertises et les imaginaires aux frontières et à la recherche de nouveaux territoires physiques ou psychiques : « Dans le paradoxe et l'ambiguïté, prend corps ce complexe d'images, de machines, de réseaux et d'intelligence comme surgirait sous la forme d'une explosion intense : un langage...? une galaxie...? un univers... ? l'embryon d'une structure vivante... ? une nouvelle frontière... ? Bref dans l'angoisse et la fascination, l'accouchement d'un Nouveau Monde ${ }^{58} »$;

2. L'extraordinaire foisonnement et les possibilités infinies qui s'ouvrent impliquent de s'appuyer sur des réseaux ouverts. Il ne s'agit pas uniquement de coopérations entre disciplines scientifiques ou filières de développement, entre les acteurs de la recherche et de l'industrie au niveau national ou au niveau international mais de mettre en place un immense système mondialisé de ressources d'informations et de réservoir d'actions : «les hautes technologies appellent par nature à la coopération et au pragmatisme. Coopération entre disciplines scientifiques, entre savoir-faire professionnels, entre entreprises ; coopération, encore, par-delà les frontières... Ainsi, de la robotique aux biotechnologies, en passant par les matériaux et les technologies de l'information, la coopération n'est pas une vaine formule, mais une nécessité vitale... L'époque technologique contemporaine s'est ouverte sous le signe des complémentarité, et non des exclusives $^{59} »$. C'est désormais dans des réseaux mondiaux que tout se joue et les programmes « Technologie, Croissance, Emploi » issus du Sommet de Versailles des Chefs d'États et de Gouvernement des Pays industrialisés en 1982 cherchent à en faire la démonstration, tout comme la recherche d'une initiative européenne dans les technologies civiles avec Eurêka et les projets qui sont associés ;

3. Le maître mot est celui de projet. Tout est projet. C'est-à-dire projection collective. À l'opposé d'une planification programmative et statique, les réseaux déploient leurs dynamiques vivantes : «Ce foisonnement et cette multitude donnent lieu à des jeux complexes d'interaction. Et c'est dans cette profusion et cette diversité qu'éclot la notion de réseaux. Les réseaux émergent et les images s'imposent. Images électroniques, car ces territoires nouveaux ne se découvrent qu'à condition d'y accéder par des formes neuves de représentations ${ }^{60} \gg$. Et ils génèrent des projets à géométries variables mobilisant des compétences multiples ;

4. Enfin, c'est bien de système dont il s'agit. Non au sens de systèmes d'armes mais au sens d'écosystème en construction dont chaque élément homme, machine, intelligence, sont interactifs et solidaires car «l'avenir appartiendra à ceux qui inventeront de nouvelles solidarités entre l'intelligence, le cour, la main des hommes, et le spectre vertigineusement évolutif des outils nouveaux ${ }^{61} »$. Une provocation assurément et une provocation pour les sciences sociales en particulier ${ }^{62}$ !

Dans cette approche active, trois questions ne sont pas oubliées : celles du risque, celle de l'éthique et celle de l'environnement.

Pour illustrer le premier point on se référera à un texte publié dans Pour une poignée d'électrons intitulé « Insécurité et prospective » qui en 20 points dresse une analyse saisissante de l'insécurité qui deviendra « le thème dominant 
de la décennie $80^{63} »$; on pourrait aussi bien dire des décennies suivantes jusqu'à aujourd'hui. L'irruption de nouveaux groupes humains n'est pas sans conséquence et tout devient possible, y compris « une guerre électronique des religions » susceptible de se déclencher à tout moment. Ce qu'il convient désormais d'intégrer dans toute prospective c'est l'ensemble des déplacements des « centres de gravitation de nos équilibres». Nulle vision béate et idéologique de la technologie mais une tentative pionnière de la penser dans sa complexité et de la penser tout en agissant.

Sur le deuxième point deux références sont à mettre sur la table : la première est inscrite dans les écrits d'Yves Stourdzé qui marquaient l'étroite relation entre choix technologiques, éthique et politique. La deuxième c'est l'organisation, en lien avec la Présidence de la République et la suite du Sommet de Versailles, de la Conférence internationale de bioéthique ${ }^{64}$ qui, bien avant les réunions internationales organisées par l'UNESCO $^{65}$, instaure une réflexion fondamentale sur les sciences de la vie.

Enfin le troisième point est lié aux travaux mis en œuvre au CESTA sur les milieux humides, sur vivre et travailler au pays en l'an 2000 et sur l'environnement en général ${ }^{66}$. René Passet, économiste était impliqué dans ces travaux ${ }^{67}$.

\section{Le CESTA, un think tank?}

Le terme « think tank » qui sert à désigner «la réunion d'un ensemble de personnes ou de ressources intellectuelles engagées dans la production d'analyses à destination des autorités publiques, des entreprises privées et institut d'en- seignement ${ }^{68} \gg$ ne correspond qu'imparfaitement aux missions et surtout aux actions du CESTA. On peut toutefois retenir ce terme car il s'agissait bien d'un ensemble de personnes d'horizons disciplinaires divers mais majoritairement issues des sciences humaines et sociales engagées à la fois dans des travaux d'analyse dans le court et moyen terme dans différents domaines scientifiques et technologiques, mais aussi de réseaux d'acteurs diversifiés nationaux et internationaux engagés dans des projets impliquant aussi bien les autorités publiques que les centre de recherche, les entreprises ou des organismes de formation.

Ce qu'il faut souligner c'est que ce caractère pionnier en France, alors qu'on fait généralement remonter les think tanks en France aux années 1990, ne s'inscrivait pas dans le type d'organisation dont Stourdzé précisément avait fait la critique et pour lequel il recherchait une nouvelle voie ; une nouvelle manière de penser des découvertes scientifiques et des développements technologiques foudroyants interpellant frontalement notre intelligence, les collectivités humaines capables de se mobiliser dans de nouveaux projets politiques planétaires et la conscience aiguë qu'il n'y a pas un temps pour l'action et un temps pour la réflexion mais que l'un et l'autre, comme dans tout mouvement révolutionnaire, sont indissolublement liés. Sa proximité du politique et sa dépendance à des financements interministériels en faisaient un instrument fragile. Son efficacité démontrée dans l'impulsion de projets européens et internationaux notamment vers le Japon et la Chine n'ont pas suffi lorsqu'un gouvernement de cohabitation s'est installé. Son assise théorique et politique issue de 68 et d'un courant libertaire l'éloignait définitivement des mœurs habituelles 
de la haute administration. Malgré un appel de pure forme à des repreneurs aucun examen sérieux des dossiers déposés visant la poursuite des travaux engagés ne fut réalisé.

\section{Conclusion}

Nous avons cherché à éclairer, sans être exhaustif et sans chercher à l'être, la place de la réflexion sur l'histoire et la prospective de l'époque et la singularité ou du moins la spécificité de la démarche entreprise par Yves Stourdzé et le CESTA et ses fondements théorico-pratiques. Ce qui caractérise cette vision du monde c'est, malgré une conscience très vive des risques, sa volonté d'ouverture et d'une certaine manière son optimisme ou encore, comme dirait Ernst Bloch, son principe d'espérance ; l'idée qu'il n'y a d'issues que dans la complémentarité et la solidarité, c'està-dire dans le collectif. Les stratégies qui émanent de cette analyse sont des stratégies d'alliance et de réseaux. C'est-à-dire des stratégies politiques et des stratégies de pouvoir.

On peut naturellement engager une critique de cette conception de la technologie et la qualifier tour à tour d'idéologique ou d'utopique et la référence même que je fais à Ernst Bloch peut y engager ; l'utopie étant, comme le soulignait Paul Ricœur, ce qui se manifeste comme une alternative critique à l'existant tandis que l'idéologie s'attache à légitimer le réel ${ }^{69}$. Or, en l'occurrence il ne s'agit ni de l'un ni de l'autre ou, plus exactement, l'ensemble de la démarche peut-être à la fois et simultanément qualifiée d'utopie planétaire et d'idéologie scientificotechnologique. Elle désigne toutefois moins un ensemble constitué, une visée à atteindre que le mouvement même qui pourrait la faire naître. L'avenir n'y est pas un simple déploiement de virtualités orientées vers le champ des possibles réalisables comme on pourrait le penser, mais bien une aventure : «Le savoir comme énigme. La formation comme cheminement. Demain, aprèsdemain comme labyrinthe... Car sous nos yeux la connaissance est en train de se transformer en jeu d'aventure ${ }^{70} »$. La connaissance comme une aventure ? Oui, mais alors celle d'un gai savoir énigmatique et mystérieux à poursuivre au long d'un labyrinthe sans fin ouvert sur une prospective aux antipodes de celles qui projettent devant nous ce parcours surdéterminé où pas à pas l'avenir se referme dans les figures imposées de la reproduction du même. Prospective, j'écris ton nom : liberté. 
$R \cdot \dot{E} \cdot F \cdot \dot{E} \cdot R \cdot E \cdot N \cdot C \cdot E \cdot S$

Laboratoire de conjoncture et prospective, Bilan des études de prospective, Février 1977.

M. BLOCH, L'Étrange défaite, témoignage écrit en 1940, Folio histoire, 2014.

G. GEUENS, « Think Tank » in Les nouveaux mots du pouvoir, abécédaire critique, sous la direction de P. Durand, Éditions Aden, 2007.

E. JANTSCH, La Prévision technologique : cadre, techniques et organisation, OCDE, Paris juin 1968.

P.-A. JULIEN, P. LAMONDE, D. LATOUCHE, « La méthode des scénarios. Une réflexion sur la démarche et la théorie de la prospective » in Travaux et Recherche de Prospective, coll. Schéma général d'aménagement de la France $\mathrm{n}^{\circ}$ 59, La Documentation française, Paris 1975.

A. LIPIETZ, H. ROUILLEAULT, Sur la pratique et le concept prospectif du matérialisme historique, Mémoire D.E.S. Paris 1972.

B. MOREL, «Conjuguer l'histoire : conjoncture et prospective » in L'Actualité économique, vol. 51, n², 1975, pp. 194-208.

P. RICEUR, L'Idéologie et l'utopie, Seuil 1997. J.P. SARTRE, "Question de méthode » in Critique de la raison dialectique, Tome 1, Paris, Gallimard, 1967.

L. SFEZ, L'Administration prospective, Paris, Armand Colin, Collection U - série science administrative, 1970.

Y. STOURDZÉ, Les Ruines du futur, Cahiers d'Utopie, 1978, réédition aux éditions Sens\&Tonka, 1998.

Y. STOURDZÉ, Organisation, anti-organisation, Mame 1973, en cours de réédition aux éditions Sens\&Tonka, 2015.
Y. STOURDZÉ, Pour une poignée d'électrons, pouvoir et communication, Paris, Fayard, 1987, Sens\&Tonka, 2016.

Les Mémoires de la Recherche État des versements. Dossier réalisé par Bénédicte ADAO, Ministère de l'Enseignement Supérieur et de la Recherche, Mission des archives, 2010 
$\mathrm{N} \cdot \mathrm{O} \cdot \mathrm{T} \cdot \mathrm{E} \cdot \mathrm{S}$

1. C'est l'enjeu notamment de Google créant Alphabet, août 2015.

2. Il faut relire les pages d'humour que Marcuse consacre aux jeux de la Rand Corporation. H. Marcuse, L'Homme unidimensionnel, Paris, Éd. de Minuit, 1969, pp. 104-107.

3. OREAM, Organismes d'études d'aménagement des aires métropolitaines.

4. L. Sfez, L'Administration prospective, Paris, Armand Colin, Collection U - série science administrative, 1970, p. 9.

5. L. Sfez, Ibid. pp. 37-43.

6. «les organes prospectifs naissent là où se développe la planification, qu'il s'agisse du Commissariat au Plan à vocation assez générale, de la DATAR (planification de l'espace) ou de la DGRST ou de l'Armée (planification d'un secteur; la recherche ou la Défense nationale» L. Sfez, Ibid. p. 264.

7. L. Sfez, Ibid, p. 73.

8. L. Sfez, Ibid., p. 212.

9. L. Sfez, Ibid., p. 219.

10. Lucien Sfez en conclut que ces «technostructures perdront de leur importance le jour où de véritables alternatives politiques seront proposées au pays. »Ibid., p. 390. En même temps, il montrera ultérieurement que les décisions finalement prises obéissent à des aléas, des pressions et des torsions qui réintroduisent dans le système des considérations ayant peu à voir avec les propositions issues de la prospective. Sfez L., Critique de la décision, Paris, Presse de la fondation nationale des sciences politiques, 1976. Voir notamment les annexes p. 353 et suivantes.

11. Analysée par Erich Jantsch, Consultant à l'OCDE «La prévision technologique : cadre, techniques, organisation », OCDE, Paris juin 1968.
12. Y. Stourdzé ajoutera bien plus tard, en 1986, le « choc nippon » comme élément déterminant déplaçant la valeur ajoutée vers les technologies grand public. Ce glissement est significatif de son approche : on surmonte les crises mais surmonte-t-on aussi facilement ce qui touche au cœur du système de décision politico-industriel ? Y. Stourdzé, « Eurêka : le Monde, l'Europe, la France », in Pour une poignée d'électrons, pouvoir et communication, Paris, Fayard, 1987, p. 414. 13. Laboratoire de conjoncture et prospective, Bilan des études de prospective, Février 1977. Référence : http://lara.inist.fr/bitstream/handle/2332/1588/XX CNE-Prospective 001379.pdf? sequence $=1$. Il s'agit d'un laboratoire privé créé et dirigé par Christian Goux, économiste, qui a soutenu en 1965 une thèse d'État sur « l'horizon prévisionnel » C. Goux, L'horizon prévisionnel, Cujas, 1969.

14. Ibid. p. 1. Plus directement Bertrand Morel qui fait partie du laboratoire dira : "la prospective cherche à mettre en place des stratégies de conquête ou de maintien de pouvoirs. Tels sont son projet, son but et par conséquent sa nécessité historique. Ils sont intimement liés à la situation économique et politique à la fin de la Seconde Guerre mondiale : rivalités entre les deux blocs politiques de l'Ouest et de l'Est, rivalités entre les différents pôles du bloc de l'Ouest pour le contrôle et l'hégémonie de l'Occident, pour l'extension du mode de production dominant aux quatre coins de la planète». B. Morel, Conjuguer l'histoire : conjoncture et prospective in L'Actualité économique, vol. $51, \mathrm{n}^{\circ} 2$, 1975, p. 195.

15. Ibid. p. 9.

16. P. Veyne, Comment on écrit l'histoire, essai d'épistémologie, Paris, Seuil, 1971.

17. J.P. Sartre, "Question de méthode » in Critique de la raison dialectique, Tome 1, Paris, Gallimard, 1967. 18. Laboratoire de conjoncture et prospective, Bilan des études de prospective, Février 1977, Opus cité p. 9. 
19. Ibid., p. 16.

20. Ibid., pp. 89-90.

21. Ibid., pp. 9, 91, 98.

22. J.P. Sartre, Opus cité, p. 96.

23. Laboratoire de conjoncture et prospective, Opus cité, pp. 17, 79, 99.

24. Ibid., pp. 73-78.

25. Le Centre d'Études des Systèmes et des Technologies Avancées, Proposition d'organisation, de fonctionnement et de mise en œuvre, rapport à Monsieur le Premier Ministre, 23 décembre 1981. Tapuscrit.

26. Ibid., pp. 3 et 4 .

27. Ibid., p. 2 et p. 5.

28. S. Nora, A. Minc, L'informatisation de la société, La documentation française, 1978. F. Gros, F. Jacob, P. Royer, Sciences de la vie et société, rapport au président de la République, La Documentation française, 1979. Pour mémoire, Joël de Rosnay qui sera un des rédacteurs du rapport pour la création du CESTA avait rédigé un document annexe au rapport sur les sciences de la vie intitulé Biotechnologies et Bio-industrie, Seuil, La Documentation française, 1979.

29. Voir l'article de Brigitte Chamak dans ce numéro des Quaderni.

30. Le Centre d'Études des Systèmes et des Technologies Avancées, rapport à monsieur le Premier ministre, Opus cité, p. 11.

31. Ibid., pp. 19 et 21.

32. Ibid., p. 26. L'OTA fut supprimé en 1995 par les Républicains. On considérait alors que son travail dupliquait celui de plusieurs ministères et agences gouvernementales. Depuis des voix s'élèvent régulièrement demandant son rétablissement

33. P. Musso, P., « Rétrospective de la prospective territoriale : de la Datar à la Diact » in Territoires 2030 n³, Août 2006.

34. Y. Stourdzé, Les Ruines du futur, Cahiers d'Utopie, 1978, réédition aux éditions Sens\&Tonka, 1998.
35. Y. Stourdzé, Organisation, anti-organisation, Mame 1973, en cours de réédition aux éditions Sens\&Tonka, 2015, p. 152.

36. Étude sur la Bretagne citée dans Pour mémoire, Actes de la journée " La prospective au sein des ministères de l'Équipement et de l'Environnement depuis les années $1960 »$, № Hors-série de la Revue des ministères de l'Égalité des territoires et du logement et de l'Écologie, du développement durable et de l'énergie, Hiver 2013, p. 45.

37. Y. Stourdzé, Les Ruines du futur, Opus cité, pp. 17, 20 et 23.

38. Y. Stourdzé, Organisation, Anti-organisation, Opus cité p. 141 «La prophétie sert ainsi de moteur à l'organisation. L'énergie rebondit d'objectifs intermédiaires en objectifs intermédiaires pour le plus grand profit de l'organisation ».

39. J. Attali, Jérémie in Dictionnaire amoureux $d u$ judaïsme. Paris, Editions Plon-Fayard, 2009, p. 256.

40. Y. Stourdzé, Les Ruines du futur, Opus cité, p. 22 et p. 30 .

41. Ibid. p. 80.

42. Y. Stourdzé, « Sphère et labyrinthe » in Pour une poignée d'électrons, Opus cité, p. 52.

43. Ibid.p. 10. Pour une analyse d'Organisation, Antiorganisation voir l'article de Franck Cormerais dans ce dossier des Quaderni.

44. Y. Stourdzé, Organisation, Anti-organisation, Opus cité, p. 135.

45. Ibid. p. 152.

46. Y. Stourdzé, Les Ruines du futur, Opus cité, p. 30. 47. Ibid. p. 65 et p. 87.

48. Voir par exemple « Contrôler le déplacement » et sa référence à la guerre de 1870, ou « autopsie d'une machine à laver » in Pour une poignée d'électrons, Opus cité.

49. M. Bloch, L'étrange défaite, témoignage écrit en 1940, Folio histoire, 2014, p.180. Cité de manière 
détournée par Yves Stourdzé à travers une citation du prospectiviste Bertrand de Jouvenel : « De la vapeur à l'électricité » in Pour une poignée d'électrons, Opus cité, p. 111.

50. Y. Stourdzé, Organisation, Anti-organisation, Opus cité, p. 56 et p. 58.

51. Y. Stourdzé, Les ruines du futur, Opus cité, p. 64. 52. Ibid., p. 94 : « La charge explosive qui git dans l'usage ».

53. Note 251/YS/VG du 14 aout 1986, Tapuscrit.

54. Y. Stourdzé, Organisation, Anti-organisation, Opus cité, p. 152.

55. La critique qui a été faite à cette démarche c'est qu'elle relevait d'une idéologie empiriste. A. Lipietz, H. Rouilleault, Sur la pratique et le concept prospectif du matérialisme historique, Mémoire D.E.S. Paris 1972, p. 119.

56. Cognitiva 85, À la frontière de l'intelligence artificielle, des sciences de la connaissance, des neurosciences, Paris 4-7 juin 1985 organisé par le CESTA, 1'AFCET et l'ARC. 2 volumes de proceedings.

57. Milieux extrêmes / Environmental extremes, Marseille 25-26 et 27 février 1986. Un volume de proceeding et un ouvrage aux éditions du CESTA : $\dot{A}$ la conquête de l'inaccessible - Recherches et technologies en milieux extrêmes, 190 pages.

58. M.A.R.I ou l'accouchement d'un Nouveau Monde. Note 193/YS/VG, 4 juin 1986, Tapuscrit.

59. Y.Stourdzé, « Eurêka : le Monde, l’Europe et la France » in Pour une poignée d'électrons, Opus cité, p. 409.

60. Note 193/YS/VG, 4 juin 1986, Opus cité.

61. Note 251/YS/VG du 14 août 1986, Tapuscrit.

62. E. Barchechath, C. Hermant, P. Rolle, M. Thonon (ed), La provocation - Hommes et machines en société, Programme de coopération internationale « acceptabilité des nouvelles technologies par le public », groupe de travail « Technologie, Croissance, Emploi », Édition
CESTA, 1985. Introduction Yves Stourdzé, p. 11. 63. Y. Stourdzé, «Insécurité et prospective » in Pour une poignée d'électrons, Opus cité pp. 209-211.

64. Conférence internationale de Bioéthique, 19-21 avril 1985, Édition CESTA, Paris 1986.

65. Son Comité International de Bioéthique (CIB) est créé en 1993.

66. Aux éditions du CESTA : Terres et eaux - Approches techniques pour conserver et mettre en valeur les zones humides ; La filière eau-assainissement en France et dans le monde; Vivre et travailler au pays en l'an 2000 - Technologies nouvelles et développement local ; Pour une politique scientifique internationale de l'environnement.

67. R. Passet, L'économique et le vivant, Payot 1979, R. Passet, La bioéconomie de la dernière chance, Les Liens qui Libèrent, 2012.

68. G. Geuens, « Think Tank » in Les nouveaux mots du pouvoir, abécédaire critique, sous la direction de P. Durand, Éditions Aden, 2007. Alain Supiot dans un article du Monde soulignait de manière humoristique qu'un « Tank » c'était aussi un char d'assaut ! A. Supiot, « Pour un droit du travail digne de ce nom », Le Monde, samedi 17 octobre 2015.

69. P. Ricœur, L'idéologie et l'utopie, Seuil 1997.

70. Note 250/YS/VG, 14 aout 1986, Tapuscrit. 


\title{
$R \cdot E ́ \cdot S \cdot U \cdot M \cdot E$
}

En 1978, dans un ouvrage intitulé Les Ruines $d u$ futur Yves Stourdzé, sociologue des organisations et de la communication, s'engage dans une réflexion prospective sur les sciences et technologies en rupture radicale avec les travaux de prospective réalisés en France depuis la fin des années 1950. Cette approche va nourrir les travaux du Centre d'Études des Systèmes et des Technologies Avancées (CESTA) qu'il dirigera de 1982 à 1986 ; un think tank atypique conçu initialement sur le modèle de l'Office of Technology Assessment (US). Dans cet article nous retracerons dans un premier temps les attendus, rôles et apories de la prospective des années 1960-1980 avant d'analyser les apports d'Yves Stourdzé et du CESTA.

\begin{abstract}
In his 1978 book, "Ruins of the future", the sociologist of organizations and of communication Yves Stourdzé made a radical break with the science and technology forecasting work carried out in France since the end of the 1950s. His approach was to guide the work of the Center for Studies of Systems and Advanced Technologies (CESTA), an atypical think tank originally modeled after the U.S. Office of Technnology Assessment, which he directed from 1982 to 1986. In this paper we will first trace the role, initial goals, and paradoxes of forecasting in France in the years 19601980, then going on to analyze the contributions of Yves Stourdzé and CESTA.
\end{abstract}

